\title{
El estudio de los significados en los procesos educativos. Un reto metodológico
}

\section{(The study of the meanings in educational processes. A methodological challenge)}

*Lya Sañudo

Coordinación de Educación Básica de la Secretaría de Educación, Jalisco - México

*Email de correspondencia: lyasa54@yahoo.com.mx

\section{RESUMEN}

La investigación educativa tiene como finalidad comprender las experiencias vividas dentro del contexto de la enseñanza, así como, dotar a los educadores con una estructura del saber que sea teórica, dinámica y compleja para que, a través de esta, los enseñantes informen a la sociedad sobre el fenómeno educativo. En este trabajo se aborda el estudio de los significados y su construcción como resultado de la reflexión-teorización de las experiencias vividas dentro del contexto del saber. Para esto, se presenta a la fenomenología y la hermenéutica como soportes de la investigación educativa en los procesos de descripción y comprensión de las situaciones que empíricamente se viven en los entornos educativos. A lo largo del trabajo, se aborda una amplia discusión sobre los significados: su importancia, conceptualización, la construcción del discurso para su documentación, así como su interpretación por medio de la fenomenología y la hermenéutica.

Palabras clave: significados; hermenéutica; fenomenología; procesos educativos; experiencias educativas.

\begin{abstract}
The educational research aims at understanding the experiences gained within the teaching context and providing educators with a structure of knowledge that is theoretical, dynamic and complex so that, through this, teachers are capable of reporting about the educational phenomenon to society. This work addresses the study of meaning and its construction as a result of reflection and theori-
\end{abstract}


zation of experiences lived inside the educational context. Thus, it presents phenomenology and hermeneutics as a support for educational research in the processes of description and understanding of situations that are empirically experienced in educational environments. Throughout the work, we address a broad discussion about the meanings: their importance, conceptualization, the construction of discourse for their documentation, and their interpretation through phenomenology and hermeneutics.

Keywords: meanings; hermeneutics; phenomenology; educational processes; educational experiences.

\section{Cumbres}




\section{INTRODUCCIÓN}

Este texto se orienta a la descripción e interpretación de las estructuras esenciales de la experiencia educativa. La fenomenología y la hermenéutica están siendo desarrolladas y aplicadas de forma productiva a la investigación educativa, sobresalen tanto como una postura epistemológica comprensiva y como una metodología cualitativa.

El valor fundamental de esta alternativa, de acuerdo a van Manem (2003), radica en su capacidad de acceder a la comprensión profunda de la experiencia humana investigada desde diversos ámbitos disciplinarios. Ha proporcionado una base para que los investigadores lleguen a describir e interpretar lo que realmente constituye educativo. Este camino supone una determinada orientación intelectual y actitudinal del investigador, enraizada en las nociones y presupuestos de la fenomenología y la hermenéutica.

Aunque no se consideran en el sentido de un conjunto de procedimientos de investigación que se pueden dominar de un modo relativamente rápido, sí se desarrollan a través de un camino, que está interesado primordialmente por el estudio del significado esencial de los fenómenos, así como por el sentido y la importancia que estos tienen. Son métodos reflexivos que pretenden recuperar, analizar, describir e interpretar comprensivamente, las estructuras esenciales de la experiencia educativa, responden a la necesidad de que la investigación educativa consolide, desde un pluralismo metodológico, una forma de construir las formas de comprender y hacer lo educativo; que impliquen una mejora real para la educación (van Manem, 2003).

Todas las acciones, situaciones y las relaciones que se viven en la educación tienen una naturaleza empírica: esencialmente práctica, relacional, ética y comunicativa, donde el lenguaje tiene un sentido trascendente. La fenomenología describe las experiencias a través de entrevistas y observaciones en la mayoría de las veces.

Pero en la investigación dedicada a la educación se aspira a obtener una comprensión profunda de la experiencia educativa, a desarrollar co- nocimiento pertinente que permita al educador contar con una estructura teórica dinámica y compleja que dé cuenta del fenómeno educativo. De acuerdo a van Manem (2003), se propone desarrollar la teoría de lo único, afrontar la unicidad de cada experiencia educativa a través de la reflexión-teorización.

Este texto está organizado en cinco apartados, el primero hace énfasis en que la educación y sus procesos son el centro de la investigación y que una manera de llegar al sentido profundo de los sujetos es la construcción de los significados; el segundo, intenta hacer una reflexión acerca de los abordajes teóricos sobre el término "significado" y los procesos implicados. El tercer apartado, breve, recupera al lenguaje como el vehículo esencial y pertinente para desplegar los significados para ser estudiados. El cuarto está centrado en los métodos fenomenológico y hermenéutico, que se plantean como los más adecuados para describir e interpretar los significados. Finalmente, el último apartado propone un acercamiento desde el análisis elemental y descriptivo hasta el interpretativo con la idea de lograr textos que se aproximen a la teorización educativa.

\section{EL SIGNIFICADO EN LA EDUCACIÓN}

La educación es una relación de personas, entre personas y sobre personas, en donde las que participan crecen, se mejoran y perfeccionan como efecto de esa relación. Puede definirse "como el sistema de acciones reflexivas, intencionadas y sistemáticas [auto] transformadoras del ser humano, en un contexto histórico determinado" (Sañudo, 2006, p. 21). En este espacio existe una intención clara y sistemática de formación, a través de una interacción compleja, donde prevalece la comunicación verbal. Pero más aún, de acuerdo a Bazdresch (2000), la relación educativa no depende solo de la interacción de los sujetos actuantes, sino de la "constitución" de dicha interacción en la acción constructiva de procesos de significación.

Los procesos de significación están "en medio" de la interacción, es decir, de la percepción 
y de la interpretación por parte de los sujetos; de contenidos y conocimiento de un sujeto frente a otro sujeto, donde uno es estudiante. La significación

... enriquece y ayuda a completar el como sucede y se efectúa la posibilidad de que un sujeto entienda a otro según los significados que el primero desea expresar, mediante el recuerdo de la significación, visualizar cómo un sujeto (educador/educada) atribuye sentido a las acciones y por tanto se entiende a sí mismo/misma, y correlativamente, de qué manera a comprender el yo del interlocutor (educando, educanda) y la conducta de los otros en general (Bazdresch, 2000, p. 35).

El constitutivo central del significado es la vivencia del sujeto por eso sus acciones tienen sentido subjetivo para él mismo en primera instancia. El interlocutor observa sólo lo que le deja ver u oír. Este sujeto puede objetivar el sentido que encontró para la acción a través del lenguaje y hacerla comunicable. "Significar para el sujeto es adquirir cada vez más alto grado de conciencia acerca de lo que vive y de sus efectos en su persona" (Bazdresch, 2000, p. 36).

Interpretar los significados del otro y darles un sentido a sus acciones, es comprender su subjetividad, y cuando este proceso es mutuo entonces se establece una interacción intersubjetiva. Facilita la auto consciencia y la comunicación en la cual el entendimiento mutuo puede darse plenamente.

Es en este plano de las expresiones subjetivas donde se identifica la acción educativa, ya que la interacción verbal cotidiana no es suficiente para generar procesos de formación. El contenido de la intersubjetividad es el significado que cada uno de los sujetos pone en juego para crear o construir de manera conjunta una significación común. Así, la significación queda entendida como un proceso reflexivo, acumulativo y progresivo opera una modificación en la estructura cognitiva de los sujetos (Sañudo, 2006).

Queda entonces en el centro mismo de lo que constituye lo educativo, los procesos de significación, en ambos agentes educativos: estudiante y docente, en el caso del trabajo en el aula, el docente de manera intencionada y sistemática plantea situaciones que le permiten al estudiante construir los significados que median entre cada uno y la comprensión del mundo en el que viven, entre cada uno y sus iguales.

La institución escolar, el currículum -como organización de las actividades-y los procesos educativos - como producto emergente del intercambio de la institución, su currículum y los educandos- son momentos de la circunstancia educativa que es situacional y determinada por las dimensiones socioculturales que lo definen (Medina, 2005).

La escuela, para decirlo de otro modo, no es sitio al que el estudiante va a "descubrir verdades", sino a construir y negociar significados. Dentro de las propias ciencias - y en las escuelas donde se enseña el conocimiento generado por aquéllas- el objetivo primordial no consiste tanto "en el establecimiento de verdades" como en la elaboración de significados que hagan la realidad comprensible y que permitan el desarrollo de una tecnología útil. El lenguaje, la música, los dibujos y diagramas, los números, los sistemas de comunicación, la forma de vestir, las computadoras, los medios de transporte, y un extenso etcétera, son todos artefactos o utensilios culturales que nos hacen comprensibles nuestro entorno y nos permiten operar sobre él (Medina, 2005, p. 9).

En el proceso educativo y especialmente en el aula, se usan instrumentos para compartir significados reconocibles y para llevar a cabo acciones o actividades con significado.

\section{ACERCAMIENTO TEÓRICO AL CONCEPTO DE SIGNIFICADO}

En este apartado se pretende un acercamiento analítico-descriptivo a partir de referentes de autores, como Saussure (1945), Pierce, Lonergan (2008), Vygotski (1995), Bruner (1988) que van desde la propuesta lingüística, hasta la psicología y la filosofía. No se trata de tratado exhaustivo, sino tiene la finalidad de recorrer diversas propuestas que brinden un panorama sustentar cómo el significado constituye una categoría re- 
levante en la investigación educativa.

El primer acercamiento al término de "significar" se remonta a la lingüística. Desde la perspectiva estructuralista, representada por Saussurre (1945), el significado es un componente conceptual indisociable de un signo lingüístico, al igual que el conjunto de fonemas articulados o significante, identifica una relación arbitraria entre ellos. El padre de la semiótica moderna Pierce, agrega "la referencia" que es la relación entre el significante y el significado; los tres elementos están absolutamente asociados, de manera que se presenta en una estructura: el triángulo semiótico (von der Walde, 1990).

Un grupo de personas pueden compartir un mismo significado, pero pueden referirse a él con diversos significantes, derivados de la cultura, como él. De la misma manera se puede usar un significante polisémico. Desde esta perspectiva, el signo representa una idea relativa de una cosa, una idea, algo imaginable o incluso inimaginable. Es por esta razón que el signo, como vehículo comunicativo genera una imagen en el que lo interpreta que nunca será idéntica a la original, puede ser alterna, más o menos elaborada, o cuando más, similar. Es el lenguaje lo que permite que dos sujetos tengan significados compartidos.

Sin dejar de lado la necesaria existencia de la triada semiótica, para estas propuestas el foco de interés, es el signo lingüístico, para los procesos educativos es más relevante el significado, ya que es este el que media entre los agentes educativos y es también el que se construye a partir de los contenidos escolares. El significado es por un lado una construcción individual, eminentemente subjetiva y por otro, presupone la interacción con los semejantes, y mientras más semejantes mayor es el marco de referencia en los significados compartidos.

Lonergan (1986) entiende el mundo que se construye, como aquel mediado por el significado, en el que "antes de actuar lo imaginamos, lo planeamos, investigamos sus posibilidades, pensamos pros y contras (...), desde el principio estamos comprometidos en actos de significación; sin ellos el proceso no ocurriría ni llegaríamos al final" (p. 3). Para este autor, realmente lo que cuenta para el sujeto y para un grupo de sujetos es lo que cada cual significa de la realidad en la que vive, ya que la realidad significada es la mediación que permite referirse al entorno. "El sujeto interpreta su mundo inmediato primero y mediato después por medio del significado; habla de él y se refiere a él por el significado" (Sañudo, 2006, p. 42).

Cuando es necesario que el sujeto se transforme o transforme su hacer, el proceso que se lleva a cabo "a través de actos intencionales que se proponen fines, elige sus medios, busca colaboradores, dirige operaciones" (Lonergan, 1986, p. 4); los significados se manifiestan en un segundo nivel, reflexivo en donde existe un nivel de conciencia de la acción, se refiere y da cuenta de ella. El significado se construye a partir de operaciones de la experiencia de la intelección y del juicio en un todo dinámico en la que, "la experiencia estimula la indagación, y la indagación no es más que la inteligencia que se pone a sí misma en acto" (Lonergan, 1967, p. 3). El autor considera a la cultura como un sistema de significación compartido y aunque no le dedica tanta profundidad considera ambas dimensiones la individual y la colectiva.

Esto presupone la interacción con otros sujetos en donde comparte significados en formas de cultura específica. En cada uno de estos grupos se concibe la "realidad" de una determinada manera y no de otra, y la representa mediante símbolos compartidos por los miembros de ese grupo cultural; por tanto, se puede suponer que los símbolos y sus significados son productos construidos históricamente y son recibidos por los nuevos miembros como parte de su herencia cultural.

La construcción y recepción de significados es el resultado de un proceso que involucra la participación activa de los sujetos en actividades compartidas, en las que se actúa de acuerdo a ciertas reglas, se imita a los demás, se construyen y se cuentan historias, se experimentan los triunfos y los fracasos comunes, etcétera; lo que conforma, a final de cuentas, la noción de pertenencia a un grupo. La construcción individual está asociada a la participación en cada grupo cultural, y es enriquecida por la experiencia di- 
recta de cada sujeto. Como ya se ha mencionado compartir significados no implica similitud o equivalencia, el lenguaje, la reflexión, la interacción y la discusión entre ellos acercará los significados entre sí, y además constituirá un marco de referencia compartido

Aquí se puede articular una propuesta que se origina en otro contexto teórico, como es el caso de la teoría desarrollada por Vygotski. El significado para Vygotski tiene una naturaleza semántica y comunicativa, que al "desdoblarse" permiten una interacción del sujeto con sí mismo constituyendo la conciencia. El significado que tenga el sujeto, el educador o el investigador educativo, media en sus acciones y la conciencia para tomar decisiones y ciertas posturas ante su labor; es decir, "la conducta humana depende de las operaciones basadas en significados" (Vygotski, 1995, p. 153). Según Vygotski, las funciones mentales superiores del ser humano deben ser consideradas como producto de una actividad mediada; en este caso el papel mediador es un instrumento de naturaleza semiótica, es decir un significado.

Los signos con los que refiere el educador su práctica se interiorizan de la cultura y a partir de un nivel de conciencia permite la conversión de los sistemas de regulación externa en autorregulación. Estos sistemas de autorregulación modifican dialécticamente la acción educativa. Mientras en la edad temprana la acción domina el desarrollo, posteriormente la acción emerge del significado "al pensar, actúa. La acción interna y externa son inseparables, la imaginación, la interpretación y voluntad son procesos internos realizados por la acción interna" (Vygotski, 1995, p. 153).

A diferencia de Lonergan, que propone un significado eminentemente interpretativo que puede inscribirse en la fenomenología, Vygotski establece como el origen del significado la interacción social y se le incluye en la dialéctica. Ambos comprenden que el significado media entre el sujeto y su entorno; también que esa relación entre la acción (actividad) y el significado, como dos manifestaciones de un solo fenómeno, permi- te la transformación, esencialmente por la vía de la conciencia y la relación de esta con el entorno -en ambos casos- y de la reflexión, en el caso de Lonergan (Sañudo, 2006, p. 43).

Por otro lado, en el ámbito educativo, Pérez (1998) explora la construcción de significados y la relaciona con la elaboración simbólica. "Esta capacidad confiere la posibilidad de representar la realidad, valorarla, modularla virtualmente, transformarla, y comunicar sus transformaciones y valoraciones" (Pérez, 1998, p. 214); describe el proceso como vital, en parte incierto, contradictorio y ambiguo. La interpretación de significados se hace de manera subjetiva y comprensiva, en un proceso de construcción común de significados. Pérez (1998) enfatiza la importancia ecológica del escenario vital del sujeto. El contexto es un escenario plural con diferentes niveles que van desde el micro al macro contexto y que conformo como una red formando un sistema. El educador hace de mediador simbólico entre los diversos planos del contexto, supone el paso de un dominio de sentido a otros. El significado es una mediación que permite al educador construir su mundo y referirse a él para reflexionar su hacer y transformarlo intencionalmente.

La pregunta es ċlos sujetos se apropian del entramado de significados presentes en su comunidad? ¿Es el sujeto el que construye sus significados y los comparte y modifica a partir de la interacción en su entorno? La intervención de Bruner (2000, p. 19) puede traer alguna claridad para esta tensión "la mente se constituye por y a la vez se materializa en el uso de la cultura humana." De acuerdo a esto, lo que constituye el significado en cada sujeto es precisamente la interacción entre su entorno cultural y su conciencia y experiencia personal. En el nivel macro, interactúa con un sistema de creencias, valores, supuestos, saberes, artefactos, etcétera; y uno micro, en donde los sujetos se mueven, deciden y actúan e interactúan. Es en este contexto sistémico en el que las personas perciben la realidad y construyen los significados que les van a permitir compartir o discrepar del al grupo. Por lo tanto, los significados son mediaciones, herra- 
mientas que posibilitan a los sujetos referirse, conocer, comprender, ordenar y actuar en su entorno; los significados orientan la acción de cada sujeto y de una comunidad en un sistema cultural concreto. El significado es un producto final y el proceso para obtener ese producto es la acción de significar.

Regresando a Lonergan, el sujeto se mueve y comprende a través de una realidad significada, es decir no es la realidad la que cuenta sino la manera en cada uno interpreta esa realidad. Ya que la realidad humana está constituida de actos de significación que van más allá de la experimentación. Lo que es significado, no sólo es experimentado, sino que también de alguna manera comprendido y comúnmente también afirmado. Desde el principio al final del proceso, estamos comprometidos en actos de significación; y sin ellos el proceso no ocurriría ni llegaríamos al final. El significado es una parte importante del vivir, las reflexiones sobre el significado y el control consecuente del significado, son aún más importantes. Los cambios sociales y culturales son en su raíz cambios de significado.

Si el propósito de una investigación es interpretar y constituir los significados en el proceso educativo, con la intención de comprender el entramado de significados en un grupo escolar, o el intercambio significativo en la interacción intersubjetiva entre docentes y estudiantes; desplegar el sentido que se encuentra en el origen de los actos o las decisiones, también puede ser posible recuperar el proceso de significación de un grupo de estudiantes con respecto a un área de conocimiento determinado. Es viable, además, conocer la red de significados que le dan sentido a la práctica docente, tanto para comprender lo que el docente piensa y a partir de ahí el entendimiento de las decisiones estratégicas que toma o la determinación de las interacciones. Y a partir de esta significación se pueden plantear modelos de resignificación para la mejora educativa.

Para desplegar estos entramados de significados, es necesario utilizar el lenguaje como el vehículo pertinente, aunque no el único. En el siguiente apartado se profundiza este elemento

\section{EL LENGUAJE COMO MEDIACION}

Hay por lo menos dos niveles de significación. El nivel primario, espontáneo, reflexivo, en el cuál no solamente empleamos el lenguaje, sino que también decimos lo que entendemos con el lenguaje. Y el segundo, es reflexivo. Los significados se originan en las sensaciones y percepciones que las personas experimentamos y básicamente son cualidades de las cosas. Por lo tanto, de acuerdo al razonamiento realizado antes, los significados son construcciones individuales y en su dimensión de pertenencia una cultura donde se comparten saberes, valores, supuestos y creencias, que ordena su mundo. En ese proceso la participación de lenguaje, es vital para desplegar, compartir y modificar los significados.

El lenguaje es en sí mismo un producto cultural que posibilita la comunicación, construir discursos, e interaccionar. se transmite de generación en generación mediante la enseñanza y el aprendizaje. Los significados permiten a las personas conocer, comprender y ordenar el mundo que les rodea y con base en ello actuar en consecuencia, de tal suerte que se puede afirmar que los significados orientan la actuación de las personas en el sistema cultural en el que están inmersos.

Como se mencionó ya, el origen de los significados es individual, en un primer momento, pero al estar mediado por el lenguaje, se convierte en compartido con el grupo social, lo que permite ampliar la información que se tiene sobre un signo. Así el lenguaje oral, escrito o gráfico además de tener una función en el proceso de significación, facilita el estudio de los significados individuales o de un conjunto de sujetos de una cultura.

\section{EL ABORDAJE METODOLÓGICO}

Es una realidad, la investigación educativa puede constituir los significados asociados a la educación de sujetos o grupos, algunos autores (van Manem, 2003; Martínez, 1989; \& Melich, 1994) proponen los métodos más adecuados están basados un abordaje fenomenológico-herme- 
néutico.

En este sentido, por ejemplo, es viable recuperar la racionalidad de la acción mediante la sistematización y a partir de ello construir los significados que le dan sentido. "La cuestión del significado no radica en buscarlo como un rasgo inmanente de las cosas, sino en reconocer que este es creado. La naturaleza no genera significado alguno, las sociedades sí. El significado de un símbolo, es decir, comprender su connotación, es una tarea que recae en el propio intérprete." (Medina, 2005, p. 1) De acuerdo al autor, el significado es un constructo, quiere decir que es el resultado de un acto interpretativo; cada relación comunicativa implica un acto de interpretación y de negociación de significados.

El significado se expresa en el lenguaje (verbal, corporal. gráfico...) orientado a otro u otros que lo interpretan de acuerdo a un momento y especio determinado; se despliega el significado a través del sujeto y tiene sentido en esa circunstancia concreta, cuando se pueden identificar los significados compartidos, entonces se puede construir el entramado de significados que constituyen una cultura. Por lo tanto, es la verbalización la que puede desplegar el significado y como puede deducirse las herramientas metodológicas para "extraer", analizar y construir el significado de un sujeto o varios deben ser aquellas que dejan registradas las expresiones de los sujetos. Los métodos pueden ser la entrevista, texto discursivo o la narración, transcrita en el caso de que sea grabada, cuando el texto se acompaña de las notas y su circunstancia, entonces se refiere a un corpus, que se encuentra listo para ser segmentado para su análisis.

Es importante destacar la importancia que tiene poner atención a la escritura dentro del ámbito fenomenológico hermenéutico, tomando en cuenta los silencios, las anécdotas como un elemento metodológico, la manera anecdótica de narrar mediando en la reflexión y en la acción, buscando mostrar algo siempre en una integración entre las partes y el todo, así como dando el seguimiento necesario a cada uno de los temas a tratar y profundizándolos.

En esta lógica el abordaje fenomenológico, se encuentra en la primera intención de la investigación, donde la descripción de un objeto-sujeto en el proceso educativo, es el propósito. Se orienta a la recuperación de la experiencia vivida y, en cambio, la hermenéutica se orienta a la como cada individuo interpreta los textos de la vida. Desde lo fenomenológico significaría investigar cuestionando como experimentamos el mundo y nuestra estancia en el mundo, es cuestionarnos por conocer lo más intrínseco del ser; así lo educativo consiste desde aquí en atender las exigencias de los fenómenos que existen o experimenta el sujeto.

La fenomenología busca conocer más profundamente la naturaleza, la experiencia dentro de la misma, no nos ofrece teoría, pero si contacto directo con el mundo, es descubrir y describir las estructuras de significado interno y de experiencia. El carácter de la fenomenología es de tipo reflexivo, y hacer fenomenología hermenéutica, es elaborar descripción interpretativa sobre algún producto (significado) o proceso educativo (significación). El investigador permanece en los hechos ya que va a aprehender el significado esencial con un contacto directo de la experiencia original vivida. Se hace la aplicación del lenguaje, reflexión de la, experiencia vivida mostrada tal cual y así, la experiencia vida implica conciencia de vida inmediata y pre reflexiva.

La hermenéutica es el método de investigación que proporciona la posibilidad de interpretar símbolos en campos de significación compleja (Sañudo 2005). Esta vía vincula la teoría y la práctica -fundamental para la teorización educativa a partir de la investigación- por medio de la reflexión, porque "únicamente la problemática de la reflexión puede venir en ayuda de un texto o un conjunto de signos susceptibles de ser considerados como texto" (Ricoeur, 1987, p. 49).

\section{LAS OPCIONES DE ANÁLISIS}

En el desarrollo de la investigación las etapas de análisis se repiten cada vez que culmina un acercamiento al campo, e manera que los resultados tienen efecto en las decisiones en los si- 
guientes acercamientos. Teniendo un corpus, es decir de un texto ampliado a partir del cual se van a construir los significados, se segmentan partes del texto que responden a campos de observables pertinentes a las categorías previstas, p. e. creencias, saberes, valores, supuestos asociados a un objeto de investigación concreto, p. e. los significados de los docentes sobre su práctica, o sobre la inclusión educativa: o los significados de ciertos contenidos históricos o cívicos de los alumnos. Algunos autores le llaman a esta fase reducción de datos, aquí se discrimina la información útil de la que no lo es. El producto es un texto fenomenológico (van Manem, 2003).

El sujeto que investiga parte de una prenoción teórica que cognitivamente le provee de lo necesario para recortar de la totalidad, aquellos observables que quiere indagar. Esta prenoción opera como unos conceptos ordenadores que "recorta y ordena" aquellos observables de la totalidad educativa. Es obvio mencionar que entre más preciso y claro sea el concepto ordenador, mayor posibilidad tiene el investigador de tomar las decisiones metodológicas pertinentes. Así, se selecciona el método que va a recuperar y a registrar "congelando" en tiempo y espacio esos observables (Sañudo, 2005, p. 9).

La diversidad del sentido no estriba en él mismo, sino en la lectura teórica e intencionada que se haga de él. Por ejemplo, en un registro de observación en donde se recuperan las evidencias de aprendizaje de los alumnos (en sentido amplio) en una situación de aula, es posible que las evidencias que se recortan del corpus difieran y por supuesto, la interpretación, también. ¿De qué depende? Pues, de que p. e. el término "aprendizaje" es conceptualizado de diferentes maneras, si lo comprendemos como construcción de significados, como apropiación de contenidos o como aprendizaje significativo. Posiblemente, se segmenten del texto de la observación evidencias más o menos similares, pero, ciertamente, la interpretación va a diferir: un segmento que evidencie una característica de aprendizaje significativo, no necesariamente implica construcción cognoscitiva o una apropiación. Según Dilthey (1998a) la diversidad del sentido puede resolverse verificando de manera intersubjetiva el sentido construido para lograr gradualmente la verdad por aproximaciones sucesivas. Después de contar con un grupo de textos descriptivos se puede iniciar con los procedimientos hermenéuticos a seguir, desde la el hecho o el texto educativa hasta la construcción de significados teóricos- Esta lógica se acerca al círculo hermenéutico de Dilthey (1998b), que es un movimiento del pensamiento que va del todo a las partes y de cada parte al todo, y de este otra vez a las partes. En términos de Morin (1984) constituiría un bucle dialógico, recursivo y hologramático, de modo que cada movimiento aumenta el nivel y la calidad de la comprensión.

Tal es el caso de situaciones concretas como la indagación intencionada del trabajo en un aula, en la que en un espacio concreto y en un lapso determinado suceden fenómenos educativos de naturaleza distinta. Ahí se encuentran el proceso individual interno de cada uno de los agentes, las acciones, la práctica, la interacción verbal y no verbal entre ellos, el manejo y lógica del contenido de trabajo, los productos de la sesión, las intenciones explícitas e implícitas de los agentes y del programa, las normas disciplinarias, los condicionantes institucionales y las organizaciones sociales entre otros. Estos fenómenos se pueden observar, recuperar o registrar con mediaciones metodológicas pertinentes como la observación o la entrevista, que en su versión escrita y ampliada se convierten en el corpus. Así, la materia prima en un proceso hermenéutico es un texto que tenga sentido (Sañudo, 2005).

Se desentraña el sentido de las expresiones lingüísticas del texto y se construye el símbolo que expresa el carácter no inmediato de la aprehensión de la realidad. Los símbolos son "las expresiones de doble o múltiple sentido cuya lectura semántica es correlativa al trabajo de interpretación que hace explícito su segundo sentido o sus sentidos múltiples" (Ricoeur, 1987, p. 15).

Por muy diversas que puedan ser las manifestaciones sensibles, poseen características comunes y regularidades que, como materia analizable y con sentido, constituyen unidades de significado. Estas conexiones representan una estructu- 
ra teórica propia del área del conocimiento, es una conexión real pero individual y, por lo tanto, subjetiva. El tipo de análisis utilizado es de tipo descriptivo con matrices de orden y de categorización, donde se construyen unidades de significado independientes (Miles \& Huberman, 1994).

La interpretación, significa la comprensión de cualquier texto que tenga un sentido, tiene que ver con entender la interpretación como una forma de hacer evidente el sentido oculto de un símbolo (Ricoeur, 1987). Según Dilthey (1998b) este proceso se inicia en los intereses de la vida práctica, y posteriormente, requiere de un distanciamiento teórico. Este análisis es elemental de manera directa y por analogía.

Esta comprensión fenomenológica hace accesibles los elementos para la reconstrucción y, posteriormente, las formas de la comprensión superior permiten conocer las conexiones de cada parte con el todo, mediante operaciones que implican la identificación de relaciones entre dos o más unidades de significado. En esta parte del proceso puede aportar la hermenéutica de Ricoeur que propone formas de desentrañar el sentido del corpus para construir la lógica del símbolo y, por ende, el sentido. La lógica está definida por el relato de la estructura (que implica relaciones) del texto, también es el conjunto de signos que organizan el texto (Ricoeur, 1987). Se pretende con esto llegar a la interpretación que "se refiere a una estructura intencional de segundo grado que supone que se ha constituido un primer sentido" (Ricoeur, 1987, p. 15). La interpretación busca la estructura común en los elementos expresados, sistematiza y teoriza aquello que conforma el corpus.

Tanto los primeros hallazgos "rupestres" y sus características, como los supuestos construidos hasta aquí, pueden ayudar a establecer los criterios para empezar a segmentar el corpus para ir desentrañando el sentido al interior del texto. ¿Qué segmentos son significativos por su reiteración? ¿Cuáles de ellos lo son por lo que representan en la lógica del texto sin importar su aparición recurrente? ¿Qué se puede construir en los diferentes eventos de los segmentos de manera inductiva? ¿Es posible hacer las prime- ras afirmaciones? Este puede ser un primer análisis elemental o categorial que permite ordenar y clasificar el contenido, para esto es útil utilizar matrices de orden y categoriales en la fase descriptiva (Miles \& Huberman, 1994).

A partir de los significados y las relaciones que se vayan constituyendo, se establece una estructura que responde a la lógica interna del mismo texto, y en última instancia, de las manifestaciones del fenómeno que se quiere comprender. La estructura permite entender la totalidad y la coherencia de los diversos elementos en función de su finalidad. El fenómeno empieza a comprenderse y las redes empiezan a desplegarse, implicando a todos los niveles de complejidad y diferentes manifestaciones o contextos que, de acuerdo a la finalidad, pueden ser interpretados como una totalidad.

Para construir las redes de significado, se utilizan las formas de comprensión superior, es decir métodos lógicos como la inducción, al análisis, la comparación y la deducción a través de matrices de análisis interpretativo o meta análisis; igualmente, no se dejan de lado las diferencias derivadas de su forma particular dentro del campo empírico. En este sentido, Ricoeur (1995) hace hincapié en la importancia del contexto social, ya que entiende los casos singulares en el todo social, el análisis estructural articula en su justo peso la influencia del ambiente en la determinación de la acción humana. Esta debe ser "objetivada" como objeto de conocimiento sin perder sus características y riqueza de significado.

La parte operativa de la interpretación considera que se ha constituido un corpus a partir de la recuperación en texto de una totalidad concreta de una realidad educativa, y esta representación de las manifestaciones educativas, son susceptibles de múltiples interpretaciones. Como menciona Ricoeur (1987), no se trata de aceptar o no que los términos sean polisémicos sino de asumir que su interpretación depende del lector, "se trata del sentido del texto, y precisamente en tanto es independiente de la intención del autor es un sistema mudo de signos puede ser construido de diversas maneras" (Ricoeur, 1987, pp. 4647). Aun aceptando en un sentido amplio que esto 
es válido, es importante traer las reflexiones de Eco (1992) que puntualiza que la interpretación no puede ser arbitraria, sino que necesariamente debe validarse en el sentido y lógica interna del texto o de los observables de la totalidad de donde se recuperó1. En este proceso, cualquier descripción se recoge a través de una experiencia vivida y, al mismo tiempo, evoca tal experiencia; es validada y valida a su vez la experiencia vivida. A esto se le llama "círculo validador de la investigación” (van Manem, 2003). Sugiere al investigador, un cuidado riguroso, regresar al corpus durante todo el proceso y, con mayor énfasis, cada vez que se construya un significado para constatar que lo construido se encuentra dentro de la lógica empírica original.

Por otro lado, el parámetro de la interpretación es el sentido de los mismos significados, ya que sólo tienen un campo limitado de posibilidades. Un significado, entonces sólo puede referirse a ese campo de posibilidades y no a otro. Si el investigador está indagando acerca de la calidad aprendizaje del estudiante en una institución y circunstancia determinada, no sería válido que interprete sobre las estrategias docentes, cuando menos no de manera directa con ese corpus.

A partir de algunos de los trabajos de Ricoeur (1976, 1987 \& 1995) y Martínez (1989) se pueden distinguir una serie de acciones posibles que apoyan el trabajo interpretativo del investigador:

- Iniciar el trabajo reflexivo a partir de un supuesto flexible y en construcción de acuerdo al avance de la investigación.

- Desarrollar empatía con el actor, intentar comprender su marco interno de referencia, entender lo más cercanamente posible el fenómeno, con toda su complejidad en su contexto cultural e histórico.

- Establecer los elementos conceptuales como "conceptos puente" necesarios para empezar a trabajar y resignificarlos en una interacción recursiva.

- Preguntar qué sentido constituye un significado razonable, en el sentido de una "buena" configuración.

- Distinguir las relaciones posibles entre los conceptos que se van construyendo que permiten transformar los supuestos y las afirmaciones hasta ahora elaboradas.

- Diseñar una siguiente mediación que permita acceder a la realidad acotada a partir de una intención más clara y focalizada cada vez.

- Construir la lógica de las relaciones de los símbolos interpretados de manera progresiva.

- Toda comprensión debe ser una mejor comprensión que la anterior, lo que implica progresión dialéctica, acumulación del sentido. La interpretación debe ser razonable y con una comprensión de mayor profundidad que la que puede permitir el sentido común.

- El texto debe comprenderse desde su interior, y desde su lógica, entender lo que el texto "dice" y su sentido implícito. El significado construido y articulado es y permanece ligado al fenómeno mismo.

- Contrastar la interpretación en construcción de las partes con el significado global del texto (o evidencia) como un todo, y posiblemente, con otra información complementaria o equivalente. Esto permitirá resultados razonables, consistentes, coherentes y sin disonancias cognoscitivas (Sañudo, 2005, p. 19).

- El intérprete articula el sentido que intencionadamente construye en el texto de manera que es relevante para su propia investigación o para la comunidad a la que está dirigida.

Establecidos los criterios anteriores, es po-

\footnotetext{
${ }^{1}$ Evidentemente, no se trata de la validación de objetividad de la epistemología positivista, sino de la construcción dialéctica que requiere el significado de un texto; sin que exista un referente en la acción y su sentido, la interpretación puede, en un descuido de rigurosidad, perder la intención de la investigación. Para profundizar en el tema es posible leer a Eco, Umberto en Los límites de la interpretación, Lumen, México, 1990
} 
sible rescatar del desprestigio una la operación cognoscitiva humana muy productiva: la intuición. A través de ella el investigador se acerca de diversas maneras al objeto de conocimiento y empieza a intuir ese sentido, igualmente, a partir de repetidas "lecturas" del corpus empieza a entender lo que ahí, en su interior, sucede. Cada vez que se produzcan estos posibles acercamientos, es necesario desde la parte, regresar al todo y viceversa, como primer momento de elaboración. Las primeras decisiones, preguntas y supuestos de los que puede partir el intérprete para realizar un análisis más sistemático y formal, pueden ser de manera muy espontánea.

Agotado un primer ciclo de análisis elemental, es posible que al establecer las regularidades, las concordancias y diferencias, las correlaciones, etc. Entre los elementos, ¿cómo se comportan cada uno de ellos en relación con los demás? ¿Cuáles son los tipos de relación que se pueden identificar? ¿Qué tipo de relaciones se pueden establecer entre las categorías (unidades de significado)? ¿Aporta a la construcción del significado el establecimiento de esas relaciones? ¿Es posible aventurar las primeras redes de significado? ¿Es posible establecer afirmaciones que implique la comprensión del sentido de los elementos y de las relaciones entre ellos? En este punto regresar tanto al paso anterior, como al corpus inicial permite revisar lo producido en relación con la lógica del todo. Esta segunda fase, interpretativa, en las diferentes construcciones donde se acumula el sentido, el investigador cuenta con los diversos modos de ordenar los significados y hacer evidentes las relaciones a través de gráficos y matrices de relaciones cruzadas, temporales, o en redes propuestas por Huberman y Miles (1994).

Cuando el investigador se aproxima a un fenómeno de estudio, tiene alguna idea provisional de su red de significados como un todo; al ser examinadas las partes -en su condición hologramática, el significado de algunas de ellas se hace parcialmente claro, y esa claridad puede aumentar al relacionar unas partes con otras y con el todo. Este proceso puede llevar a una reevaluación del significado del todo que, a su vez puede guiar a una nueva comprensión de los componentes, en un nuevo nivel interpretativo.

Así este es un proceso de un constante "ir y venir", donde nada está acabado o plenamente seguro, donde la incertidumbre forma parte del trabajo diario, donde, al fin, los constructos que se produzcan tienen una vigencia relativa. Sin embargo, todas estas consideraciones son propias de la vida humana y, por tanto, una ciencia constituida fundamentalmente de la comprensión de las acciones humanas, puede responder de manera más cercana y familiar a las preguntas propias de su naturaleza, preguntas acerca del ser humano siempre inacabado (Sañudo, 2005, p. 20).

Finalmente, cualquier investigador que decida incursionar por este tan poco claro camino podrá tener la extraordinaria experiencia de descubrir y compartir formas nuevas de acercarse al campo educativo. No es fácil tratar de construir de manera rigurosa un campo teórico nuevo, y menos lo es cuando la manera en que se propone hacerlo está en proceso también.

\section{REFERENCIAS BIBLIOGRÁFICAS}

Bazdresch, M. (2000). Vivir la educación, trasformar la práctica. Jalisco: Texto Educar/ SEJ.

Bengoa, J. (1992). De Heidegger a Habermas: Hermenéutica y fundamentación última en la filosofía Contemporánea. Barcelona: Herder.

Bruner, J. (1988-2000). Realidad y mundos posibles: Los actos de la imaginación que dan sentido a la experiencia. Barcelona: Gedisa.

Bruner, J. (2000). La educación, puerta de la cultura. Madrid: Visor.

Calvo, T., \& Ávila, R. (1991). Paul Ricoeur: Los caminos de la interpretación. Barcelona: Anthropos.

Carr, W. (1996). Una teoría para la educación: Hacia una investigación educativa crítica. Ma- 
drid: Ediciones Morata.

Delgado, J., \& Gutiérrez, J. (1995). Métodos y técnicas cualitativas en investigación en Ciencias Sociales. Madrid: Síntesis Psicología.

Dilthey, W. (1998a). Obras, VII. "El mundo histórico (La comprensión de otras personas y sus manifestaciones de vida)". En M. Jarquín (Comp.), La verdad desde sí mismo. Tres paradigmas en la investigación cualitativa (Cap. IX). México: Euterpe.

Dilthey, W. (1998b). Obras, VII. El mundo histórico (Hermenéutica). En M. Jarquín (Comp.), La verdad desde sí mismo. Tres paradigmas en la investigación cualitativa (Cap. X). México: Euterpe.

Eco, U. (1992). Los límites de la interpretación. México: Lumen.

Gadamer, H. (1977). Verdad y Método, tomos I y II. Salamanca: Sígueme.

Gadamer, H-G. (2001). Diccionario de Hermenéutica de Ortiz-Osés y Lanceros (Coords.). Bilbao: Universidad de Deusto.

Gil, J. (1994). Análisis de datos cualitativos: Aplicaciones a la investigación educativa, Barcelona: PPU.

Kemmis, S. (1996). La teoría de la práctica educativa. En W. Carr (Ed.), Una teoría para la educación. Hacia una investigación educativa crítica (pp. 17-38). Madrid: Ediciones Morata.

Lieberman, A., \& Miller, L. (2003). La indagación, como base de formación del profesorado y la mejora de la educación. Barcelona: Octaedro.

Lonergan, B. (1986). Dimensiones de significado: Mecanografiado. Guadalajara: ITESO.

Lonergan, B. (2008). Conocimiento y aprendizaje. México: Universidad Iberoamericana.
Mardones, J. (1988). Filosofía de las ciencias humanas y sociales: Materiales para una fundamentación científica. México: Fontamara.

Martínez, M. (1989). El método hermenéutico-dialéctico. En M. Martínez, Comportamiento Humano: Nuevos métodos en investigación (capítulo VII). México: Trillas.

Medina, A. (2005). La naturaleza narrativa de la mente y de la Pedagogía. Recuperado de http://www.quadernsdigitals.net/datos_web/ hemeroteca/r_24/nr_278/a_3602/3602.htm

Melich, J. C. (1994). Del extraño al cómplice: La educación en la vida cotidiana. Barcelona: Anthropos.

Miles, M., \& Huberman, A. (1994). Qualitative data analysis. USA: Sage Publications.

Morin, E. (1984). Ciencia con conciencia. Barcelona: Anthropos.

Pérez, A. (1998). La cultura escolar en la sociedad neoliberal. Madrid: Editorial Morata.

Piaget, J. (1988). Naturaleza y métodos de la epistemología, tomo 1. México: Paidós.

Piaget, J. (1991). Seis estudios de psicología. España: Editorial Labor.

Ricoeur, P. (1976). Exégesis y hermenéutica. Madrid: Cristiandad.

Ricoeur, P. (1987). Freud: Una interpretación de la cultura. México: Siglo XXI.

Ricoeur, P. (1995). Teoría de la interpretación: Discurso y excedente del sentido. México: Siglo XXI.

Sacristán, G., \& Pérez, A. (1996). Comprender y transformar la enseñanza. Madrid: Ediciones Morata. 
Sañudo, L. (2005). Hacia la definición de la teoría educativa: Posibilidades desde la hermenéutica. Revista Red de Posgrados en Educación, México. Recuperado de http://www.redposgrados.org.mx/red_de_posgrados_en_educacion/publicaciones/Revista_2.pdf

Sañudo, L. (2006). El proceso de la significación de la práctica como sistema complejo. En R. Perales, La significación de la práctica educativa (pp. 19-54). México: Paidós.

Saussure, F. (1945). Curso de lingüística general. Buenos Aires, Argentina: Editorial Losada.

Savater, F. (2003). El valor de elegir. México, D.F: Editorial Planeta.

Trías, E. (1994). La edad del espíritu. Barcelona: Destino.

van Manem, M. (2003). Investigación educativa y experiencia vivida: Ciencia humana para una pedagogía de la acción y la sensibilidad. Barcelona: Idea Books.

von der Walde, L. (1990). Aproximación a la semiótica de Charles S. Peirce. Acciones Textuales, Revista de Teoría y Análisis, 1(2), 88-113.

Vygotsky, L. (1995). Pensamiento y lenguaje. Barcelona: Paidós.

Zemelman, H. (1992). Los horizontes de la razón: El uso crítico de la teoría I. Barcelona / México: Anthropos / El Colegio de México.

\section{Cumbres}

